\title{
What is the optimal distribution of myelin along a single axon?
}

\author{
Darragh M. Walsh ${ }^{1}$, Kerry A. Landman, Barry D. Hughes \\ School of Mathematics and Statistics, University of Melbourne, Victoria 3010, Australia
}

\begin{abstract}
The myelin sheath that insulates some axons in the central nervous system allows for faster signal conduction. Previously, axons were thought to be either unmyelinated or fully myelinated. Recent experimental work has discovered a new pattern of myelination (intermittent myelination) along axons in the mouse brain, in which long unmyelinated axon segments are followed by myelinated segments of comparable length. We use a computational model to explore how myelin distribution (in particular intermittent myelination) affects conduction velocity. We find that although fully myelinated axons minimize conduction velocity, varying the spatial distribution of a fixed amount of myelin along a partially myelinated axon leads to considerable variation in the conduction velocity for action potentials. Whether sodium ion channel number or sodium ion channel density is held constant as the area of the unmyelinated segments increases has a strong influence on the optimal pattern of myelin and the conduction velocity.
\end{abstract}

Keywords: Intermittent myelination, Ion channel, Central Nervous System.

\section{Introduction}

Some axons in the vertebrate nervous system are wrapped with layers of myelin, which insulate these axons allowing for faster conduction of action potentials. The myelin sheath is produced by Schwann cells in the peripheral nervous system and oligodendrocytes in the central nervous system (CNS) $[18,19]$. Unlike Schwann cells, which act on single peripheral axons, oligodendrocytes in the CNS ensheath up to 50 axons [7], allowing them to exert influence on neural processing on a larger scale. An individual oligodendrocyte, or a cluster of neighbouring oligodendrocytes, can have a large number of nearby axons available to myelinate, but experimental data [7] supports the proposition that the choice of which axons to myelinate is not made at random [27]. Moreover, myelination has been shown to be a dynamic process that responds to environmental cues $[14,6]$.

In a recent study, Tomassy et al. [25] analysed high-resolution maps of myelination by tracing

Email addresses: darragh.walsh@unimelb.edu.au (Darragh M. Walsh), kerryl@unimelb.edu.au (Kerry A.

Landman), barrydh@unimelb.edu.au (Barry D. Hughes)

${ }^{1}$ Corresponding author Telephone +61383440584 , Fax $+61383444599$ high-throughput electron microscopy reconstructions of single axons of pyramidal neurons in the mouse brain. Analysing neurons in layers II/III from a publicly available dataset of a region of the mouse visual cortex [2,3], they observed a new pattern of myelination. Historically, axons were thought to be either fully myelinated or unmyelinated. However, when tracing neurons in layers II/III, Tomassy et al. found that 17 out of 22 neurons displayed a pattern of myelination in which myelinated axon segments are interspersed with long unmyelinated segments, and they called this newly identified pattern "intermittent myelination" (IM). In this myelination pattern, unmyelinated sections of these axons were observed to be up to 55 $\mu \mathrm{m}$ long, much longer than typical nodes of Ranvier (approximately $1 \mu \mathrm{m}$ long).

Since the discovery of intermittent myelination of (excitatory) pyramidal neurons by Tomassy et al. [25], Micheva et al. [15] also observed that the distribution of myelin in layer II/III inhibitory neurons was "patchy" with myelinated segments preferentially located near the cell body. Tomassy et al. [25] noted that neurons in layer II/III are involved in more complex cortical functioning than those found in layers V and VI, where IM was not 
observed. This raises the possibility that IM may be facilitating more complex neural functioning.

Whilst the evidence that signalling between electrically active axons and oligodendrocyte progenitor cells provides an important cue for inducing OPCs to differentiate into myelinating oligodendrocytes is firmly established, see [16], and the evidence that myelin provides more than just insulation to maximise conduction velocity $(\mathrm{CV})$ continues to mount [9], the mechanisms controlling active myelination remain largely unknown (reviewed in Snaidero and Simons [23]).

The discovery of IM by Tomassy et al. raises several questions. What is the purpose of IM? Does an IM distribution provide any advantages to signal propagation over a fully myelinated pattern? Obviously, the shortest conduction time will be for a fully myelinated axon. However, it is not obvious which distribution of myelin will maximise CV when only a fixed fraction of the length of an axon is to be myelinated. In this instance, the distribution of ion channels also becomes important.

We examine the consequences for $\mathrm{CV}$ of the partial myelination of axons. The geometry of our model depends crucially on one parameter, $L$, which is the length of both the myelinated segments and the unmyelinated segments. This pair is repeated periodically over a fixed length of axon. We only study the effects of myelin distribution on signal CV but we note that there are several other facets of neural information processing that depend on myelin distribution, such as the reliability of action potential (AP) propagation [8] and the energy consumption $[11,20,21]$.

\section{Materials and methods}

Our modelling was based on the NEURON [4, 5] implementation of a highly influential model of spike initiation in a myelinated mammalian axon by Mainen et al. [12]. We simplify this model by removing the dendrites but keep the ion channel kinetics characteristics, based on recordings of neocortical pyramidal neurons from the rat brain. Mainen et al. [12] used this kinetic model to simulate experimental measurements of rat pyramidal neurons [24].

Our simplification of this model consisted of a single axon, with inner diameter $d=1 \mu \mathrm{m}$, which is approximately twice the diameter of the axons examined by Tomassy et al. [25], and outer diameter (inner axon plus myelin lamellae) of $1.5 \mu \mathrm{m}$.
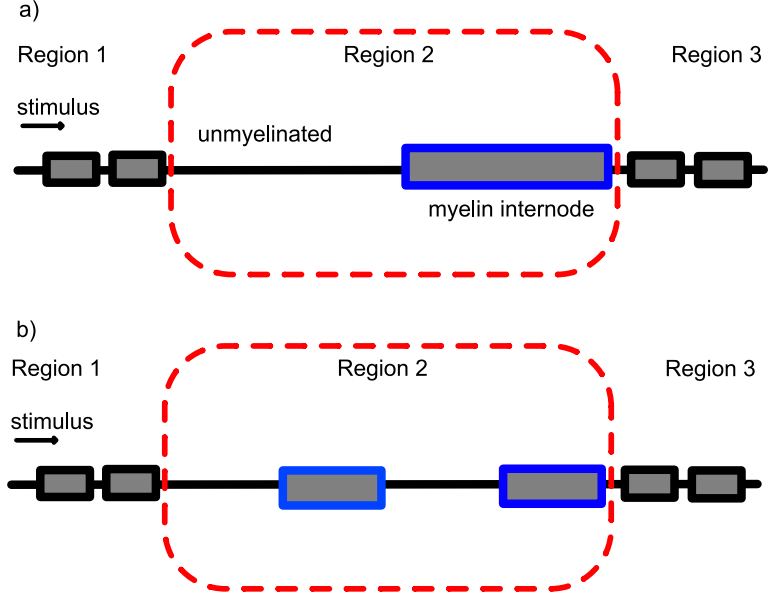

Figure 1: Schematic displaying the spatial setup of the IM simulation model. The left boundary of the axon is stimulated by a pulse which travels along a typical periodic nodeinternode region (Region 1) before reaching Region 2, which is the analysis region, where IM distribution is varied and conduction time (CT), measured in ms, is calculated. This region is $4000 \mu \mathrm{m}$ in length (so conduction velocity $\mathrm{CV}=4$ / $\mathrm{CT} \mathrm{m} / \mathrm{s}$. For illustration, panel (a) displays an intermittent myelination pattern corresponding to $L=2000 \mu \mathrm{m}$, whilst panel (b) displays an intermittent myelination pattern corresponding to $L=1000 \mu \mathrm{m}$ (in our analysis we never consider an intermittent pattern with $L>400 \mu \mathrm{m}$ ). Region 3 is identical to Region 1 and is included to avoid spurious boundary effects in the calculation of $\mathrm{CV}$.

This yields a realistic ratio of internal to external axon diameter, the g-ratio, of 0.66 . In Mainen et al. [12], internodes of length $100 \mu \mathrm{m}$ were periodically interrupted by nodes of Ranvier of length 1 $\mu \mathrm{m}$. The resting potential of the axon was taken to be $-70 \mathrm{mV}$. At the nodes of Ranvier, modified Hodgkin-Huxley (HH) type ion channels were present.

To minimize any boundary effects, we broke the axon into three regions (as shown in Figure 1): a pre-analysis region (Region 1), an analysis region (Region 2) and a post-analysis region (Region 3). The current is injected at the left boundary of Region 1. To create Region 1, we generated a sequence of 20 node-myelin pairs, each pair with combined length $100 \mu \mathrm{m}$ (a $99 \mu \mathrm{m}$ internode and a $1 \mu \mathrm{m}$ node of Ranvier). Region 1 is joined to Region 2, a 4000 $\mu \mathrm{m}$ long axon segment made up of (a variable number of) node--myelin pairs of variable length. Region 2 is the analysis region where we calculate the $\mathrm{CV}$, and is joined to Region 3, which is created in the same way as Region 1.

The uniformity of Regions 1 and 3, across all our models, facilitates cross-model comparison by en- 
suring each model has identical initial conditions whilst maintaining accurate results (by calculating $\mathrm{CV}$ in Region 2, far from the outer boundaries). If the IM pattern in Region 2 is replicated in Region 3 only a slight numerical change in conduction time (CT) results. A schematic of this setup is shown in Figure 1. Note that Region 2 is much longer than the axons traced by Tomassy et al. [25]. This was necessary to distinguish signal arrival times to high precision. It must be emphasized that we do not attempt to build a replica of the short thin axons examined in Tomassy et al. [25] but rather simulate their salient features (an IM pattern for thin pyramidal neurons). This guided our choice to use the model of Mainen et al. [12].

The left boundary node is stimulated by a $40 \mathrm{nA}$ pulse, of $0.1 \mathrm{~ms}$ duration, which initiates an AP which travels to the right along the axon. The CT is calculated by recording the time taken, in ms, for the AP to depolarize the beginning of the twentieth node to a voltage of $-15 \mathrm{mV}$, which we call $t_{1}$, and the time taken to depolarize a spatial point 4000 $\mu \mathrm{m}$ upstream of node 20 , to a voltage of $-15 \mathrm{mV}$, $t_{2}$ say. The CT over this interval is then simply $\left(t_{2}-t_{1}\right) \mathrm{ms}$, and $\mathrm{CV}=4 / \mathrm{CT}$ (in units of $\mathrm{m} / \mathrm{s}$ ).

Modelling axons displaying IM, such as the axons analyzed by Tomassy et al. [25], presents a unique challenge. The usual periodic pattern of short (approximately $1 \mu \mathrm{m}$ ) nodes of Ranvier (with a high density of sodium channels that ensures depolarization), followed by a much longer myelin internode (typically one hundred times the length of the node) is replaced by a pattern of unmyelinated and myelinated regions of comparable length. In myelinated axons, potassium channels are mostly found beneath the myelin sheath (more specifically in the juxta-paranodal region that is separated from the node of Ranvier by the paranode). Since we are not modelling repetitive firing of APs, it is assumed that sodium channel kinetics dominate AP conduction. We compare the CVs obtained by varying the IM pattern characteristic length $L$ under two sodium ion channel assumptions.

The main reason for the increased CV of myelinated axons is that it reduces effective membrane capacitance [26]. Following Mainen et al. [12], the myelin sheath is characterized by increasing the outer diameter, reducing the specific membrane capacitance $C_{m}$ and increasing the membrane resistance $R_{m}$ (both of which are independent of geometry). These values are presented in Table 1 . The relative simplicity of this representation of the myelin internodes facilitates the inclusion of sodium ion channels in the internodal regions, reflecting experimental evidence [26]. Internodal channels have been omitted from more detailed models of myelinated axons, such as [13].

Our first model assumes that the density of the sodium ion channels is constant as the length of the unmyelinated segments $L$ is increased. Our second model assumes that the number of sodium channels in the unmyelinated segments is constant (i.e. that it matches the number present in the $L=1$ $\mu \mathrm{m}$ node of Ranvier). For simplicity, we achieved this by making the density of the ion channels in the unmyelinated segments inversely proportional to the length of the unmyelinated segments $(L)$. This yields a uniform distribution of sodium channels, with the longest unmyelinated segments having lowest (uniform) sodium channel density. A noninactivating potassium current was included in the IM unmyelinated axon segments (with constant maximum conductance $g_{K}$, as described in [12]). This had a repolarising effect on APs but very little numerical effect on CT.

Spatial discretization is implemented in NEURON by dividing each each compartment (section) into $n$ segments with evenly spaced boundaries at intervals of $1 / n$. The spatial discretization was chosen to be approximately $n=1$ per micron in Region 2 where CT is calculated (see Figure 1) and also in Regions 1 and 3. A fixed time-step representing $\triangle t=0.01 \mathrm{~ms}$ was chosen and the modified Crank-Nicolson method within NEURON was used to perform the time-integration of the cable equations. To increase accuracy, interpolation was used to approximate the times at which the voltage rises to $-15 \mathrm{mV}$ at the beginning and end of Region 2, times $t_{1}$ and $t_{2}$ respectively.

We choose an IM pattern where half of the 4000 $\mu \mathrm{m}$ axon in our analysis region is to be covered with myelin and that unmyelinated segments are to have the same length as the myelinated segments. We vary the length of these segments, and hence the number of unmyelinated segment-myelinated segment pairs, and calculate the $\mathrm{CV}$ of an AP crossing this $4000 \mu \mathrm{m}$ length of axon.

\section{Results}

As noted earlier, little is known about the ion channel densities in the extended unmyelinated segments of IM patterned axons. Whilst the axon initial segment and hillock are known to have a high 


$\begin{array}{lcc} & \text { Segment type } & \text { Value } \\ R_{a}(\mathrm{ohm} \mathrm{cm}) & \text { "node" / unmyelinated } & 200 \\ & \text { internode } & 200 \\ R_{m}\left(\mathrm{ohm} \mathrm{cm} \mathrm{cm}^{2}\right) & \text { "node" / unmyelinated } & 2133 \\ & \text { internode } & 40000 \\ C_{m}\left(\mu \mathrm{F} / \mathrm{cm}^{2}\right) & \text { "node" / unmyelinated } & 0.75 \\ & \text { internode } & 0.04 \\ g_{\mathrm{Na}}\left(\mathrm{pS} / \mu \mathrm{m}^{2}\right) & \text { "node" / unmyelinated } 30000 \times H \\ (\text { sodium } & \text { internode } & 30 \\ \text { conductance }) & & \\ g_{\mathrm{K}}\left(\mathrm{pS} / \mu \mathrm{m}^{2}\right) & \text { unmyelinated } & 60 \\ (\text { potassium } & \text { elsewhere } & 0 \\ \text { conductance }) & & \end{array}$

Table 1: Electrophysiological parameters for our model axons. The two departures from the model of Mainen et al. [12] are that $R_{m}$ has been increased to $2133 \mathrm{ohm} \mathrm{cm}^{2}$ (chosen so that the time constants in the myelinated and unmyelinated sections are identical). This change was necessary to ensure the membrane resistance was suitable for an extended unmyelinated segment of the axon. The second departure is that the channel distribution is altered by introducing the factor $H$ to uniformly redistribute the sodium ion channels in the unmyelinated segments. Either the number of sodium ion channels per nodal area is held constant (in which case $H=\pi / A$, where $A=\pi d L$ is the nodal area, $d$ is the axon diameter and $L$ is the length of the node/unmyelinated segment ) or the sodium ion channel density is held constant (where a sensitivity analysis was performed with the factor $H$ chosen to yield $g_{\mathrm{Na}}=300,600$ and $\left.900 \mathrm{pS} / \mu \mathrm{m}^{2}\right)$. The nodes of Ranvier in the model of Mainen et al. [12] were $1 \mu \mathrm{m}$ in length, whereas in the IM patterns observed in Tomassy et al. [25] and in our analysis, they are extended. To capture repolarisation of APs, a constant potassium ion channel density was included in all IM unmyelinated segments (with $\left.g_{K}=60 \mathrm{pS} / \mu \mathrm{m}^{2}\right)$.

density of channels, the unmyelinated segments discovered by Tomassy et al. [25] extend much further from the soma, highlighting the need for new measurements before a definitive understanding of any biological advantage conferred by IM can be attained. However, our computational modeling enables the implications of distinct scenarios to be explored.

Each scenario is parameterized by the common length $L$ of the unmyelinated segment and of the myelinated segment in the $4000 \mu \mathrm{m}$ long Region 2 and by whether the sodium ion channel density in the unmyelinated segments depends on $L$ or not. When assuming constant sodium channel density as $L$ increases, we display results for three values of the maximum sodium conductance $g_{\mathrm{Na}}$. The results of these NEURON simulations are displayed in Table 2 and their spatial profile at time $t=4 \mathrm{~ms}$ is plotted in Figure 2.

We see from Table 2 that the optimal pattern of
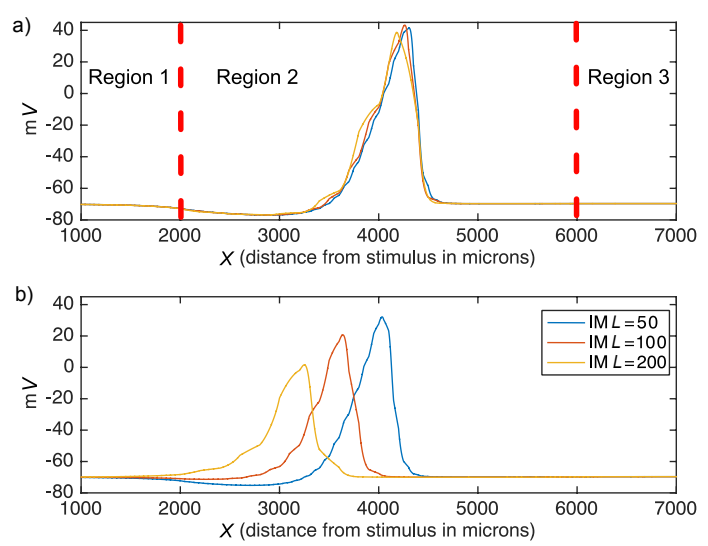

Figure 2: Comparing the effect on AP propagation of holding sodium channel density or sodium channel number per node constant as the length of the unmyelinated segments increases. Three distinct IM patterns at time $t=4 \mathrm{~ms}$ are shown in each panel. A particular IM pattern is distinguished by the common length $L$ of its myelinated and unmyelinated segments (cf. Figure 1). The density of potassium channels is held constant throughout. Computations were performed with NEURON software $[4,5]$. a) The APs resulting from three distinct periodic IM patterns (IM $L=50,100$, or $200 \mu \mathrm{m}$ ) whose unmyelinated segments have constant sodium channel density $\left(g_{N a}=900 \mathrm{pS} /\left(\mu \mathrm{m}^{2}\right)\right)$. b) The APs resulting from three distinct periodic IM patterns (IM $L=50,100$, or $200 \mu \mathrm{m}$ ), with a constant number of sodium channels, $2000 \pi$, distributed in the unmyelinated segments of each pattern, as described in Table 1.

myelin to cover $50 \%$ of an axonal region depends critically on the number of sodium ion channels in the unmyelinated segments. When the number of sodium channels in a node of Ranvier is conserved as the length $L$ of the unmyelinated segment increases, shorter internodes (and unmyelinated segments) are optimal. This is because shorter unmyelinated segments will have a much higher axonal sodium channel density than longer segments whose sodium channels must spread out over a greater distance. The opposite is true when the sodium channel density is held constant at the node of Ranvier value as $L$ increases. When the sodium channel density yields a maximum sodium conductance of $g_{\mathrm{Na}}=600$ or $900 \mathrm{pS} / \mu \mathrm{m}^{2}$, the CV has a minimum value at $L=100 \mu \mathrm{m}$. We show in Table S2 in the Supplementary Material that this is due to the presence of the sodium channels under the myelin sheath exerting a greater influence on $\mathrm{CV}$ for longer internodes, where the AP is in greater need of regeneration. When there are no sodium channels in the myelinated regions, $\mathrm{CV}$ is monotonically de- 
Constant number of channels per node

Standard
Unmyelinated
IM $L=25$
IM $L=50$
IM $L=100$
IM $L=200$
IM $L=400$

2.86

0.78

0.66

0.54

0.43

$\mathrm{PF}$
Constant channel density $\left(\mathrm{pS} / \mu \mathrm{m}^{2}\right)$

$g_{\mathrm{Na}}=300 \quad g_{\mathrm{Na}}=600 \quad g_{\mathrm{Na}}=900$

$\begin{array}{lll}0.30 & 0.38 & 0.43 \\ 0.54 & 0.66 & 0.73 \\ 0.54 & 0.66 & 0.73 \\ 0.54 & 0.65 & 0.72 \\ 0.55 & 0.66 & 0.73 \\ 0.56 & 0.67 & 0.74\end{array}$

Table 2: Simulation results comparing conduction velocity (CV) in $\mathrm{m} / \mathrm{s}$ for a standard myelination pattern $(1 \mu \mathrm{m}$ nodes, separated by $99 \mu \mathrm{m}$ internodes), a totally unmyelinated axon with diameter $1 \mu \mathrm{m}$ and five periodic patterns of IM (alternating myelinated and unmyelinated axon segments of equal length $L \mu \mathrm{m}$ ). Unmyelinated axon segments have diameter $1 \mu \mathrm{m}$, whereas myelinated segments have diameter $1.5 \mu \mathrm{m}$ ). The unmyelinated tract is a bare axon modeled by the modified Hodgkin-Huxley dynamics described in Mainen et al. [12]. Scenarios are distinguished by the ion channel model (whether channel number per node or density is held constant with increasing $L$ ). If the ion channel density is held constant, three values for $g_{\mathrm{Na}}$ are implemented. Propagation failure $(\mathrm{PF})$ occurred if the electrophysiological parameters were too weak to facilitate the propagation of the AP into the Region 2. For reference, typical CVs for pyramidal neurons in the cortex are in the range 0.2 to $4.5 \mathrm{~m} / \mathrm{s}$ for myelinated axons and 0.2 to $1.45 \mathrm{~m} / \mathrm{s}$ for unmyelinated axons, reviewed in [10].

creasing as $L$ increases.

We performed a sensitivity analysis of our results by analyzing a more detailed model of the myelin sheath developed by McIntyre et al. [13], which explicitly includes the myelin lamella and the paranodal regions where the lamella interface with the bare unmyelinated nodal segments and obtained identical conclusions regarding the critical role of the distribution of sodium channels in determining $\mathrm{CV}$ in partially myelinated axons.

\section{Discussion}

Our study reveals considerable differences in CV, with consequent implications on optimal allocation of a fixed total amount of myelin per neuron to maximise $\mathrm{CV}$, depending on the density of sodium channels in the unmyelinated segments. If we assume that the number of sodium channels in the unmyelinated regions remains constant as the common length $L$ of the myelinated and unmyelinated increases, we find that lower values of $L$ lead to faster conduction. If instead, we assume that the density of sodium channels remains constant with increasing $L$, then higher values of $L$ lead to slightly faster conduction. Thus we see that nodal characteristics can play an important role in modulating CV contrary to established theory and experiments based on axons with short nodes of Ranvier. In Table S2 in the Supplementary Material it is shown that if the sodium channels in myelinated segments are removed, then lower values of $L$ will also yield higher $\mathrm{CV}$.
The sensitivity of $\mathrm{CV}$ to the number of sodium channels in the unmyelinated segments of IM patterns that we have demonstrated in Figure 2, should be compared to the relative insensitivity of fully myelinated patterns to increases in the area of nodes of Ranvier [17], where the negative effect of increased capacitance and positive effect of an increased number of channels present on CV effectively cancelled out. In Figure S2 in the Supplementary Material we see that, holding the nodal surface area fixed, ion channel distribution can have a strong influence on which myelination pattern length gives fastest conduction. The optimal pattern length that maximises $\mathrm{CV}$ is much longer when the total number of sodium channels in Region 2 is constant compared to when the number of channels per node of Ranvier is constant.

In a recent combination of experimental investigation and simulation modelling, ArancibiaCárcamo et al. [1] found evidence consistent with ion channel densities being constant over a range of node lengths of approximately $0.5-3.5 \mu \mathrm{m}$ in rat optic nerve and cortical axons. Their modelling predicted that these changes in the length of the nodes of Ranvier could alter conduction velocities by approximately $20 \%$. The unmyelinated portions of the IM patterns that motivated our study were much longer (at approximately $50 \mu \mathrm{m}$ ). Keeping the ion channel density constant at the high levels found in nodes of Ranvier over such a large surface area seems unlikely and awaits experimental measurement.

Evidence for the dynamic redistribution of ion channels over time has been found from recordings 
of APs, which propagate (continuously) through demyelinated axons. Upon remyelination, the ion channels redistribute into the standard nodeinternode pattern to allow the recovery of saltatory conduction [10, 26]. Since the remyelinating internodes are shorter than the internodes they replace [26], recovering saltatory conduction requires distributing ion channels to create nodes of Ranvier at locations previously wrapped with myelin. It is therefore crucial that non-uniform (and dynamic) distributions of ion channels are considered. We consider the effect on $\mathrm{CV}$ of two non-uniform distributions of sodium ion channels in the unmyelinated segments in Table S1 in the Supplementary Materials.

Our results lead us to question the purpose of IM or partial myelination of axons more generally. Harris and Attwell [11] analysed the energy savings from myelinating an axon and concluded that there is not necessarily a net energy efficiency benefit when the energy required to maintain oligodendrocytes at their resting potential is included. Whilst the IM patterns and ion channel distributions we modelled can result in almost doubling the $\mathrm{CV}$, the magnitude of the differences is still small. Micheva et al. [15] noted that such small increases in CV are unlikely to be the main benefit of myelinating an axon. Such small adjustments to the CV over the short distances observed in the cortex are more likely to be important in the synchronisation of high-frequency spike-timing or the provision of metabolic support by oligodendrocytes to the axon $[10,15]$.

Support for the role of myelination in synchronising spike arrival times may be found in experiments analyzing the avian auditory system that have revealed a role for myelin in signal isochronicity. An axon was found to branch to ipsilateral and contralateral destinations in the brainstem. The contralateral path was twice as long as the ipsilateral path but the axon diameter and myelin sheath are smaller and shorter thereby halving [22] the conduction time (CT) and facilitating isochronicity.

Explaining the functional significance of partial myelination is important as it may be used to gauge how deeply oligodendrocytes are involved in orchestrating neural functioning and plasticity. Once a segment of myelin has been laid down, no synapses to neighbouring axons can originate from that location. Having extended unmyelinated regions along axons could therefore facilitate enhanced neural interconnectedness and plasticity (inspection of Fig.
2 (B) in Tomassy et al. [25] reveals synapses connected to the unmyelinated axon segments of an intermittently myelinated axon that would not have been possible had this axon been fully myelinated). However, since there are no measurements of the electrophysiological parameters of the mice axons studied by Tomassy et al. [25], our results emphasize that judgement on the functional significance of the IM pattern must be deferred until electrophysiological measurements reveal the ion channel distribution in the unmyelinated segments.

\section{Acknowledgements}

This work was supported by the Australian Research Council (DP140100339). DMW wishes to thank Dr. James Osborne for programming advice, Dr. Tania Kameneva for suggesting additional references and especially Dr. Tobias Merson for helpful comments.

\section{Appendix A. Supplementary material}

Supplementary material associated with this article can be found, in the online version, at http://dx.doi.org/10.1016/j.neulet. 2017 . 08.037 (and is also appended to this preprint).

\section{References}

[1] Arancibia-Cárcamo, I. L., M. C. Ford, L. Cossell, K. Ishida, K. Tohyama, and D. Attwell. Node of Ranvier length as a potential regulator of myelinated axon conduction speed. eLife 6: e23329, 2017.

[2] Bock, D.D., Allen Lee, W.C., Kerlin, A.M., Andermann, M.L., Hood, G., Wetzel, A.W., Yurgenson, S., Soucy, E.R., Kim, H.S., and Reid, R.C. Network anatomy and in vivo physiology of visual cortical neurons. Nature. 471(7337): 177-182, 2011.

[3] The dataset of Bock et al. 2011 containing the volume from the mouse visual cortex where IM was observed by Tomassy et al. [25] is available at www.openconnectomeproject.org.

[4] Hines, M. and Carnevale, T. The NEURON simulation environment. Neural Comput. 9: 1179-1209, 1997. Available at https://www.neuron.yale.edu/neuron/static/ papers/nc97/nsimenv.pdf.

[5] Carnevale, T. and M. Hines. The NEURON Book. Cambridge, Cambridge University Press, 2005.

[6] Chang, K. J., S. A. Redmond, and J. R. Chan. Remodeling myelination: implications for mechanisms of neural plasticity. Nat. Neurosci. 19:190-197, 2016.

[7] Dumas, L., C. Heitz-Marchaland, S. Fouquet, U. Suter, J. Livet, C. Moreau-Fauvarque, and A. Chedotal. Multicolor analysis of oligodendrocyte morphology, interactions, and development with Brainbow. Glia 63: 699-717, 2015. 
[8] Faisal, A. A. and S. B. Laughlin. Stochastic simulations on the reliability of action potential propagation in thin axons. PLoS Comp. Biol. 3 (5) e79, 2007.

[9] Fields, R. D. Myelin - more than insulation. Science 344:264-266, 2014.

[10] Freeman S.A., Desmazires A., Fricker D., Lubetzki C., and Sol-Foulon, N. Mechanisms of sodium channel clustering and its influence on axonal impulse conduction. Cell Mol Life Sci. 2016; 73 (4): 723-35.

[11] Harris, J. J. and Attwell, D. The energetics of CNS white matter. J Neurosci 32(1): 356-371, 2012.

[12] Mainen, Z. F., J. Joerges, J. R. Huguenard, and T.J. Sejnowski. A model of spike initiation in neocortical pyramidal neurons. Neuron 15:1427-1439, 1995. The source code is available in ModelDB at http://modeldb.yale. edu/8210.

[13] McIntyre, C.C., Richardson, A.G., and Grill, W.M Modeling the excitability of mammalian nerve fibers: Influence of afterpotentials on the recovery cycle. J Neurophysiol. 87: 995-1006, 2002. The source code is available in ModelDB at http://modeldb.yale.edu/3810.

[14] McKenzie, I.A., D. Ohayon, H. Li, J. P. de Faria, B. Emery, K. Tohyama, and W. D. Richardson. Motor skill learning requires active central myelination. Science 346:318-322, 2014

[15] Micheva, K. D., D. Wolman, B. D. Mensh, E. Pax, J. Buchanan, S. J. Smith, and D. D. Bock. A large fraction of neocortical myelin ensheathes axons of local inhibitory neurons. elife 5: e15784, 2016.

[16] Mitew, S., Y. L. Xing, and T. D. Merson. Axonal activity-dependent myelination in development: insights for myelin repair. J Chem. Neuroanat. 76:2-8, 2016.

[17] Moore, J. W., R. W. Joyner, M. H. Brill, S. G. Waxman, and M. Najar-Joa. Simulations of conduction in uniform myelinated fibers. Relative sensitivity to changes in nodal and internodal parameters. Biophys. J 21: 147-160, 1978.

[18] Nave, K. A. and H. B. Werner. Myelination of the nervous system: mechanisms and functions. Annu. Rev. Cell Dev. Biol. 30:503-33, 2014.

[19] Nicholls, J. G., A. R. Martin, B. G. Wallace, and P. A. Fuchs. From Neuron to Brain. 4th edition. Sunderland, Sinauer, 2001.

[20] Perge, J. A., K. Koch, R. Miller, P. Sterling, and V. Balasubramanian. How the optic nerve allocates space, energy capacity, and information. J. Neurosci. 29:79177928, 2009.

[21] Perge, J. A., J. E. Niven, E. Mugnaini, V. Balasubramanian, and P. Sterling. Why do axons differ in calibre? J. Neurosci. 32:626-638, 2012.

[22] Seidl, A. H., E. W. Rubel, and D. M. Harris, Mechanisms for adjusting interaural time differences to achieve binaural coincidence detection. J. Neurosci. 30, 70-80, 2010

[23] Snaidero, N. and M. Simons. The logistics of myelin biogenesis in the central nervous system. Glia. In press (DOI: $10.1002 /$ glia.23116), 2017.

[24] Stuart, G. J. and B. Sakmann. Active propagation of somatic action potentials into neocortical pyramidal cell dendrites. Nature 367: 69-72, 1994.

[25] Tomassy, G. S., D. R. Berger, H. H. Chen, N. Kasthuri, K. J. Hayworth, A. Vermicelli, H. S. Seung, J. W. Lightman, and P. Arlotta. Distinct profiles of myelin distribution along single axons of pyramidal neurons in the neocortex. Science 344:319-324, 2014.

[26] Vabnick, I. and P. Shrager. Ion channel redistribution and function during development of the myelinated axon. J. Neurobiol. 37:80-96, 1998.

[27] Walsh, D.M., T. D. Merson, K. A. Landman, and B. D. Hughes. Evidence for cooperative selection of axons for myelination by adjacent oligodendrocytes in the optic nerve. PLoS ONE 11 (11): e0165673, 2016. 


\section{What is the optimal distribution of myelin along a single axon? \\ Darragh M. Walsh, Kerry A. Landman and Barry D. Hughes}

Supplementary Material

Non-uniform distribution of channels in unmyelinated segments of length $L$ in an IM

pattern:

In the main text our simulations examined the effects on CV of varying how the high density of sodium ion channels present in a node of Ranvier are distributed as the node length $L$ increases. Both possibilities (constant channel number and constant channel density) resulted in a uniform distribution of sodium channels in the unmyelinated segment. We now consider two non-uniform distributions of the sodium channels; the hill and pit distributions are described below and displayed graphically in Figure S1.

In the hill-shaped distribution of ion channels, the number of channels rises linearly from half its concentration in the reference uniform distribution scenario to 1.25 times its value in the uniform scenario over a distance of $L / 3$. It remains at this concentration for a distance of $L / 3$ before falling linearly over a distance of $L / 3$ to half its concentration in the uniform distribution scenario.

Similarly, in the pit-shaped distribution scenario, the ion channel concentration falls linearly from twice its value in the reference uniform distribution scenario to half its value, then remains at this value for a distance of $L / 3$, then rises to its previous value of twice the reference uniform concentration.

When an axon becomes demyelinated, the distribution of sodium ion channels will more closely resemble the pit-shaped distribution than the uniform or hill-shaped distribution. Since the new myelin internodes generated through remyelination of an axon are almost always shorter than the one they replace [28], understanding the dynamics of ion channel distribution is crucial to understanding demyelinating diseases and stimulating remyelination. 


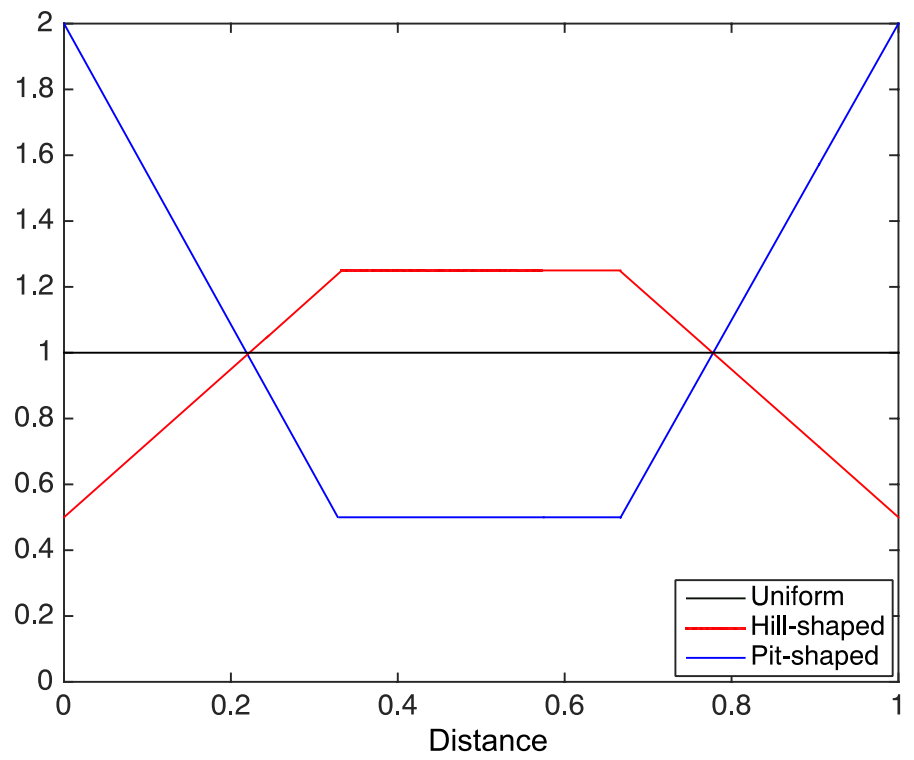

Figure S1. Three sodium ion distribution scenarios. Distance is in units of $L$, the length of the unmyelinated segments, and the vertical axis is in units of the maximum sodium conductance of the uniform distribution for the constant number of sodium channels present in the unmyelinated segment.

A)

\begin{tabular}{l|cc|cc|cc|} 
& \multicolumn{2}{c}{ Uniform } & \multicolumn{3}{c}{ Hill } \\
& CT & CV & CT & CV & CT & CV \\
\hline IM $L=25$ & 5.48 & 0.73 & 5.49 & 0.73 & 5.46 & 0.73 \\
IM $L=50$ & 5.50 & 0.73 & 5.54 & 0.72 & 5.45 & 0.73 \\
IM $L=100$ & 5.52 & 0.72 & 5.61 & 0.71 & 5.45 & 0.73 \\
IM $L=200$ & 5.49 & 0.73 & 5.61 & 0.71 & 5.51 & 0.73 \\
IM $L=400$ & 5.40 & 0.74 & 5.49 & 0.73 & 5.55 & 0.72
\end{tabular}

B)

\begin{tabular}{l|cc|cc|cc|} 
& \multicolumn{2}{c}{ Uniform } & \multicolumn{2}{c}{ Hill } & \multicolumn{2}{c}{ Pit } \\
& CT & CV & CT & CV & CT & CV \\
\hline IM $L=25$ & 5.11 & 0.78 & 5.12 & 0.78 & 5.01 & 0.80 \\
IM $L=50$ & 6.10 & 0.66 & 6.14 & 0.65 & 6.05 & 0.66 \\
IM $L=100$ & 7.44 & 0.54 & 7.52 & 0.53 & 7.37 & 0.54 \\
IM $L=200$ & 9.27 & 0.43 & 9.33 & 0.43 & 9.66 & 0.41 \\
IM $L=400$ & PF & PF & PF & PF & PF & PF
\end{tabular}

Table S1. CT (in $\mathrm{ms}$ ) and CV (in $\mathrm{m} / \mathrm{s}$ ) obtained from redistributing the sodium ion channels. A) Redistributing sodium ion channels from the scenario with constant channel density (the reference uniform scenario is from Table 2 with $\mathrm{g}_{\mathrm{Na}}=900 \mathrm{pS} / \mu \mathrm{m}^{2}$ ). B) Redistributing sodium ion channels from the scenario with constant channel number per node (the reference uniform scenario is from the first column of Table 2 with $2000 \pi$ sodium channels in each unmyelinated segment). 
Sodium ion channels beneath the myelin in an IM pattern:

\begin{tabular}{|l|c|c|c|c|c|}
\hline $\begin{array}{l}\text { g }_{\mathrm{Na}} \text { in myelinated } \\
\text { regions }\left(\mathrm{pS} / \mu \mathrm{m}^{2}\right)\end{array}$ & 30 & 0 & 90 & $\begin{array}{c}\text { Conserved } \\
\text { A }\end{array}$ & $\begin{array}{c}\text { Conserved } \\
\mathrm{B}\end{array}$ \\
\hline $\mathrm{IM} L=25$ & 5.48 & 5.55 & 5.34 & 5.28 & 4.9 \\
$\mathrm{IM} L=50$ & 5.50 & 5.59 & 5.33 & 5.41 & 5.14 \\
$\mathrm{IM} L=100$ & 5.52 & 5.68 & 5.29 & 5.52 & 5.29 \\
$\mathrm{IM} L=200$ & 5.49 & 5.84 & 5.20 & 5.63 & 5.39 \\
$\mathrm{IM} L=400$ & 5.40 & 6.29 & 5.11 & 5.81 & 5.48 \\
\hline
\end{tabular}

Table S2. The effect of sodium ion channels in the myelinated regions of an IM pattern on CT. We analyse the scenario in Table 2 with $\mathrm{g}_{\mathrm{Na}}=900 \mathrm{pS} / \mu^{2}{ }^{2}$ where, counterintuitively, a minimum CT was observed when IM $L=100 \mu \mathrm{m}$ (included as column 1). In column 2, the sodium ion channels in the myelinated segments are removed and a monotonic dependence on $L$ is recovered. By tripling the constant density of sodium channels in myelinated regions, the $\mathrm{CT}$ becomes monotonically decreasing with increasing $L$, column 3 . If the number of sodium channels in each myelinated segment is conserved (in the Conserved A and B columns, the number of channels is conserved at the value present in the $L=100 \mu \mathrm{m}$ internode with constant density $\mathrm{g}_{\mathrm{Na}}=30 \mathrm{pS} / \mu \mathrm{m}^{2}$ and $\mathrm{g}_{\mathrm{Na}}=90 \mathrm{pS} / \mu \mathrm{m}^{2}$, respectively), then $\mathrm{CT}$ is monotonically increasing as $L$ increases. 
In Figure S2 we compare the effect of varying internode length, and hence the number of nodes of Ranvier, in a standard fully myelinated pattern, keeping either the total number of sodium channels constant over all of Region 2, Figure S2 (a), or keeping the number of sodium channels per $1 \mu \mathrm{m}$ length node of Ranvier constant, Figure S2 (b). The area of the node is fixed in each case and we include sodium channels in intermodal regions with a constant maximal conductance $\mathrm{g}_{\mathrm{Na}}=30 \mathrm{pS} / \mathrm{\mu m}^{2}$

We examined the effect of redistributing all the sodium channels from a periodic $100 \mu \mathrm{m}$ standard myelinated axonal pattern spanning Region 2 ( 40 nodes of Ranvier, each with either $1250 \pi, 2000 \pi$ or $2750 \pi$ sodium channels per node), amongst the nodes of Ranvier of a different pattern length spanning Region 2 (different number of nodes), where the total number of sodium channels was kept constant. For example, if the number of sodium channels per node in a $100 \mu \mathrm{m}$ pattern is $2000 \pi$, then there will be $4000 \pi$ sodium channels in each of the 20 nodes of Ranvier of a $200 \mu \mathrm{m}$ pattern spanning Region 2.

With fixed nodal area, the 'flat maximum' in CV as pattern length increases, noted by Huxley and Stämpfli (1949) [29], doesn't materialize if the number of channels per node of Ranvier is fixed as pattern length increases, Figure $\mathrm{S} 2$ (b).

a)

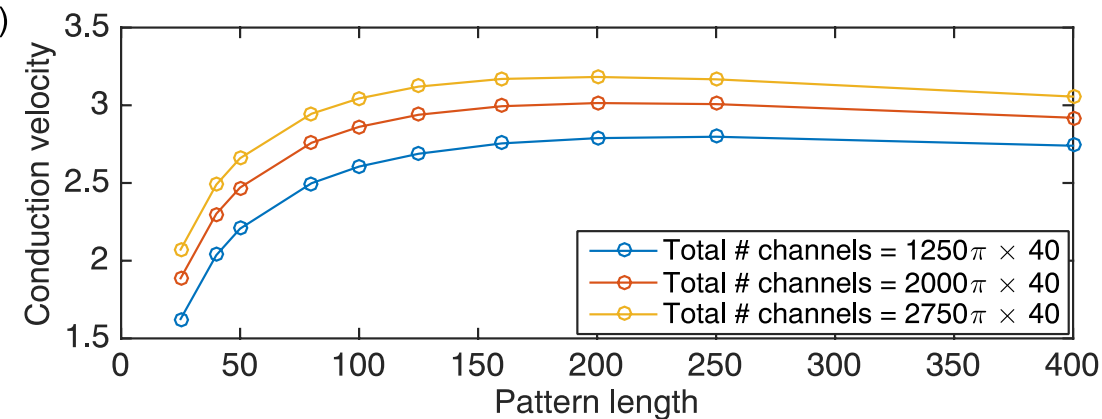

b)

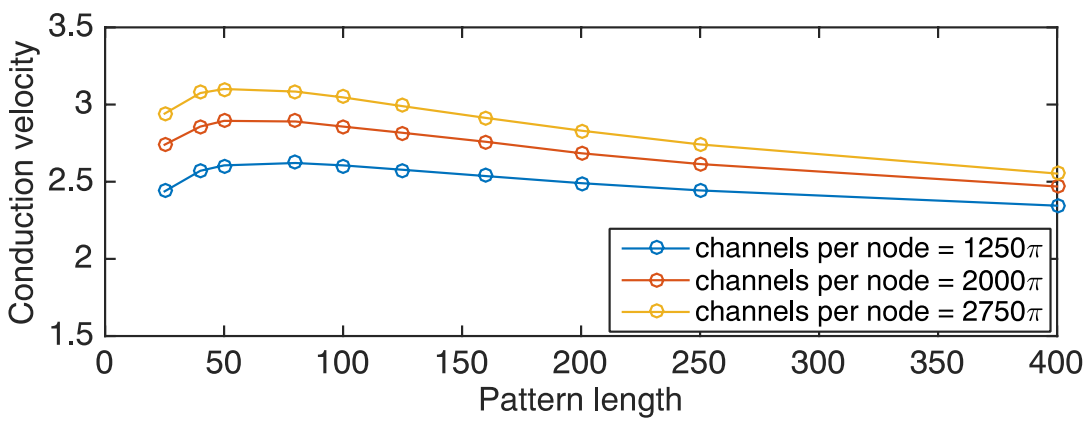

Figure S2. The effect of sodium channel distribution on CV $(\mathrm{m} / \mathrm{s})$ over a $4000 \mu \mathrm{m}$ axon (Region 2 ) in a standard, fully myelinated pattern (pattern length = internode length + node length, where node length is fixed at $1 \mu \mathrm{m}$ ) as the internode length is varied. Computations were performed with NEURON software [4, 5]. a) Dependence of CV on internode length when the total number of sodium channels in Region 2 is constant. A sensitivity analysis on the total number of sodium channels in Region 2 is included, see the text for details. b) Dependence of $\mathrm{CV}$ on internode length when, instead, the number of sodium channels in each node is constant. 
Additional references:

28. Young, K.M., Psachoulia, K., Tripathi, R.B., Dunn, S., Cossell, L., Attwell, D., Tohyama, K., and Richardson, W.D. Oligodendrocyte dynamics in the healthy adult CNS: evidence for myelin remodeling. Neuron. 77, 873-885, 2013.

29. Huxley A. F. and Stämpfli, R. Evidence for saltatory conduction in peripheral myelinated nerve fibres. J Physiol. 108(3):315-339, 1949. 